\title{
SÍNTESE DE POLIURETANOS MODIFICADOS POR ÓLEO DE MAMONA EMPREGADOS EM MATERIAIS ENERGÉTICOS
}

Roberta Jachura Rocha, José Eduardo Salgueiro Lima, Susane Ribeiro Gomes, Koshun Iha e José Atílio Fritz Fidel Rocco* Departamento de Química, Instituto Tecnológico de Aeronáutica, 12228-900 São José dos Campos - SP, Brasil

Recebido em 8/8/12; aceito em 7/1/13; publicado na web em 21/3/13

\begin{abstract}
SYNTHESIS OF MODIFIED POLYURETHANES BASED ON CASTOR OIL EMPLOYED IN ENERGETIC MATERIALS. The aim of this work was to synthesize a polyurethane polymer matrix using castor oil as a polymer chain modifier, whose characteristics can be adjusted for use as a binder in the manufacture of energetic materials such as propellant and pyrotechnics for aerospace use. We attempted the partial substitution of hydroxyl-terminated polybutadiene (HTPB), a pre-polymer commonly used as a starting polyol in obtaining energetic matrix composites. Thermoanalytical techniques were employed to characterize the material based on castor oil and the unmodified HTPB. The results showed similar behaviors, confirming the possibility of their use as polymer matrix composites through the proposed adaptations.
\end{abstract}

Keywords: hidroxyl terminated polybutadiene; castor oil; energetic materials.

\section{INTRODUÇÃO}

Um grão propelente sólido empregado em motores-foguete deve apresentar características balísticas adequadas e comportamento mecânico pré-definido em projeto, o que impõe o emprego de matérias-primas e processos de fabricação específicos para a formulação do combustível em função do projeto do motor.

As formulações de propelentes e combustíveis sólidos do tipo compósito caracterizam-se como elastômeros altamente carregados, aglomerados por um binder polimérico. ${ }^{1} \mathrm{O}$ binder, ou matriz elastomérica, é a fase contínua que aglomera as partículas sólidas de metal combustível e oxidante. Além disso, fornece as propriedades mecânicas adequadas para que o grão resultante seja capaz de resistir às solicitações mecânicas e térmicas durante as fases de vôo, transporte, manuseio e combustão. É importante o binder apresentar afinidade com as partes constituintes do motor-foguete, tais como proteção térmica e os componentes metálicos. ${ }^{2}$

Para a obtenção do binder, utiliza-se o poliol, que é uma resina de alta massa molar. Atualmente, o principal poliol (ou pré-polímero) de partida usado na obtenção da matriz polimérica de combustíveis e propelentes sólidos de motores-foguete e artefatos militares $^{2}$ é o polibutadieno líquido hidroxilado $(\mathrm{PBLH})$, cuja fórmula estrutural é $\left(\mathrm{CH}_{2}-\mathrm{CH}=\mathrm{CH}-\mathrm{CH}_{2}-\mathrm{CH}_{2}\right)_{\mathrm{n}}(\mathrm{OH})_{2}$. Esse poliol possui a característica de suportar altos carregamentos de materiais particulados sem alterar significativamente suas propriedades mecânicas originais. Além disso, o elastômero nele baseado apresenta $\mathrm{T}_{\mathrm{g}}$ igual a $-55^{\circ} \mathrm{C},{ }^{3,4}$ compatível com as condições de operação às quais o grão pode vir a encontrar durante a trajetória do vôo de motores propulsores.

No Brasil, resinas do tipo PBLH eram produzidas pela Petroflex S/A sob as marcas Liquiflex, Liquiflex He Liquiflex P. O tipo utilizado para aplicações em propelentes e combustíveis sólidos era o Liquiflex $\mathrm{H}$, devido ao seu maior teor de hidroxila, o que possibilita o aumento de ligações cruzadas por exigir uma maior quantidade de diisocianato. Porém, a produção de PBLH no Brasil foi descontinuada, tornando necessária a síntese de um poliol alternativo como material estratégico que atenda ao mercado nacional. Com isso, buscou-se a formulação

*e-mail: friz@ita.br adequada na tecnologia de pré-polímeros de uretana que ofereça um substituto parcial do PBLH.

Na manufatura de poliuretanos é possível a utilização de diversos polióis. Um composto natural que vem se destacando entre os polióis existentes, bastante utilizado na síntese de polímeros entrecruzados devido à presença de grupos hidroxilas em sua estrutura, é o óleo de mamona, que representa uma vantagem em termos de fonte renovável do poliol.

No século 16, a mamona foi importada da Índia a fim de se obter um óleo de custo inferior ao de baleia, usado como combustível de lamparinas na época. O Brasil se tornou o maior produtor mundial da mamona devido à sua fácil adaptação, e seu cultivo é feito principalmente nas lavouras do nordeste do país devido à sua resistência a longos períodos de estiagem, tornando-se de grande importância para a economia local. ${ }^{5-7}$

O óleo de mamona é um líquido amarelo, viscoso e de forte odor, comumente obtido por prensagem direta da semente ou extração com solvente. Na fabricação de poliuretanos, vem sendo usado em diversas aplicações como lubrificantes e óleos de processo, matéria-prima para combustíveis, espumas poliméricas, revestimentos e tintas, ${ }^{8-10}$ na indústria cosmética e farmacêutica ${ }^{11,12}$ e na medicina com a confecção de próteses de ossos com reduzida rejeição. ${ }^{13,14}$ Além disso, o poliuretano obtido a partir da mamona possui a vantagem de ser um produto biodegradável. ${ }^{7}$ A Índia foi a nação pioneira em utilizá-lo na obtenção do grão propelente sólido como um poliol alternativo ao usual PBLH. ${ }^{15}$ Porém, até o momento, não há disponíveis na literatura estudos desenvolvidos no Brasil acerca do emprego do óleo de mamona na formulação de propelentes para uso aeroespacial.

Quimicamente, o óleo de mamona é um triglicerídeo e aproximadamente $90 \%$ da massa do ácido graxo que constitui sua molécula é ácido ricinoléico $\left(\mathrm{R}-\left(\mathrm{CH}_{2}\right)_{7}-\mathrm{CH}=\mathrm{CH}-\mathrm{CH}_{2}-\mathrm{CHOH}-\left(\mathrm{CH}_{2}\right)_{5}-\mathrm{CH}_{3}\right)$, o qual apresenta um grupamento hidroxila secundário que pode ser empregado diretamente como matéria-prima alternativa. ${ }^{16}$ Os $10 \%$ restantes são ácidos graxos não hidroxilados, como ácidos oléicos $(3,1$ a $5,9 \%)$ e linoléicos $(2,9$ a $6,5 \%) .{ }^{17} \mathrm{O}$ grupo hidroxila presente na ricinoleína confere ao óleo de mamona a propriedade exclusiva da solubilidade em álcool, possibilitando o uso do álcool como combustível sem causar grandes danos aos motores à explosão. ${ }^{6}$ A funcionalidade do óleo de mamona é de 2,7, o que proporciona 
reações cruzadas e aumento na rigidez do polímero obtido. O valor de hidroxilas médio do óleo de mamona é de $163 \mathrm{mg}$ de KOH/g com grande pureza, recomendado para uso em poliuretanos. ${ }^{13}$

Com isso, este trabalho buscou adequar o poliol baseado em óleo de mamona para a síntese de um elastômero com as características necessárias para compor a matriz polimérica do combustível/ propelente sólido. Para a caracterização dos binders formulados, fez-se o uso da análise térmica, ${ }^{18}$ ferramenta amplamente utilizada nos estudos dos mecanismos de combustão e decomposição térmica de compósitos ${ }^{19}$ e explosivos. ${ }^{20}$ Essa análise é útil no trato de propelentes e combustíveis formulados com base em PBLH e outros pré-polímeros, devido à estrutura física heterogênea desses compósitos. Foram utilizadas as técnicas de termogravimetria (TG), calorimetria exploratória diferencial (DSC) e a análise térmica dinâmico-mecânica (DMTA).

A análise DMTA permite a obtenção de vários parâmetros, sendo um deles a relação (E”/E') entre o módulo elástico ou de módulo de armazenamento (E'), e o viscoso ou módulo de perda (E"), conhecido como $\tan \delta$ (ou fator de perda), cujo valor máximo está relacionado com a temperatura de transição vítrea, Tg. $\mathrm{O} \tan \delta$ também se caracteriza como índice de viscoelasticidade e capacidade de amortecimento, ${ }^{21}$ que fornece informações sobre as contribuições relativas dos componentes elástico e viscoso do material viscoelástico. ${ }^{2} \mathrm{Os}$ materiais que apresentem valores de $\tan \delta$ menores do que 1 exibem comportamento mais elástico e, em geral, módulo de armazenamento relativamente mais alto. ${ }^{22}$

O comportamento viscoelástico de polímeros possibilita a caracterização de seu desempenho mecânico nas condições apropriadas de tempo e temperatura. Em baixas temperaturas, o material polimérico apresenta um comportamento vítreo caracterizado por sua rigidez. Na região vítrea, seu módulo não varia de forma significativa com a temperatura ou tempo, pois sua energia térmica não é suficiente para promover qualquer translação/rotação de segmentos na cadeia polimérica. Com o aumento da temperatura, pode-se observar a transição vítrea $\left(\mathrm{T}_{\mathrm{g}}\right)$, caracterizada por uma expressiva mudança nas propriedades mecânicas do polímero (o módulo de armazenamento dos elastômeros, em alguns casos, pode ser reduzido em três vezes). Essa mudança pode ser atribuída ao movimento dos segmentos poliméricos que geram o "amolecimento" do polímero e, consequentemente, uma redução no módulo de armazenamento (E') e aumento no módulo de perda (E"). ${ }^{23}$ Por fim, em uma região de temperatura ainda mais alta, o polímero adquire o comportamento de borracha. Os domínios das transições vítreas e borrachosas caracterizam o comportamento viscoelástico do polímero e determinam a aplicabilidade dos elastômeros.

Foram selecionadas as formulações dos elastômeros de poliuretanos com base em óleo de mamona tendo em vista comparar seus resultados aos de matrizes poliméricas que possuem o PBLH como base. O processo de obtenção pode ser realizado em uma etapa ou em duas etapas de síntese.

\section{PARTE EXPERIMENTAL}

Os elastômeros de poliuretano podem ser sintetizados de duas formas: pelo processo de uma etapa ("one shot"), ou pelo processo de duas etapas, também conhecido como de pré-polímeros. Neste trabalho, utilizaram-se as duas formas. Em geral, a via de uma etapa é a mais utilizada na formulação de propelentes e combustíveis sólidos, porém, o processo em duas etapas gera uma cadeia polimérica mais bem organizada em termos de propriedades, principalmente mecânicas, devido ao pré-arranjo gerado na primeira etapa com a formação do pré-polímero. Para um melhor entendimento, os próximos itens descrevem ambas as vias de obtenção.

\section{Sistemas de uma etapa ("One-Shot")}

Um dos meios de obtenção de elastômeros de poliuretanos é pela reação de um diisocianato-tolueno (TDI) ou diisocianato-difenil-metano (MDI) com um poliol e um extensor de cadeia (diol ou amina) numa única etapa, denominada "one-shot". Esse método é efetivo com o TDI, devido a sua baixa reatividade. A adição do MDI proporciona que a reação ocorra de forma muito rápida, o que dificulta seu controle. Além disso, o MDI puro é sólido e deve ser aquecido, criando um empecilho a mais no processo. A exotermia da reação nos processos de uma etapa é alta, podendo ser controlada acrescentando-se cargas minerais que absorvam parte da energia liberada. As reatividades dos compostos hidroxilados (polióis e dióis) devem ser similares às dos diisocianatos ou poliisocianatos. O Esquema 1 mostra o sistema de uma etapa.

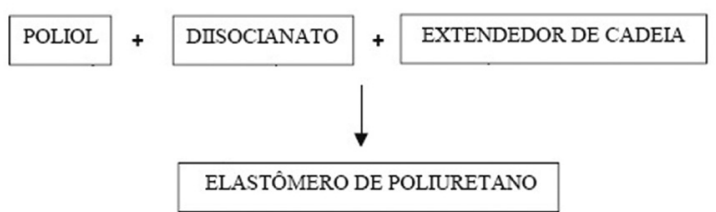

Esquema 1. Obtenção de elastômeros de poliuretano através do sistema de uma etapa

Os elastômeros de poliuretano obtidos pelo sistema de uma etapa podem ser estendidos através de dois tipos de extensores de cadeia: um diol de cadeia curta com baixa massa molar ou uma amina primária, conforme mostrado nos Esquemas 2 e 3, respectivamente.

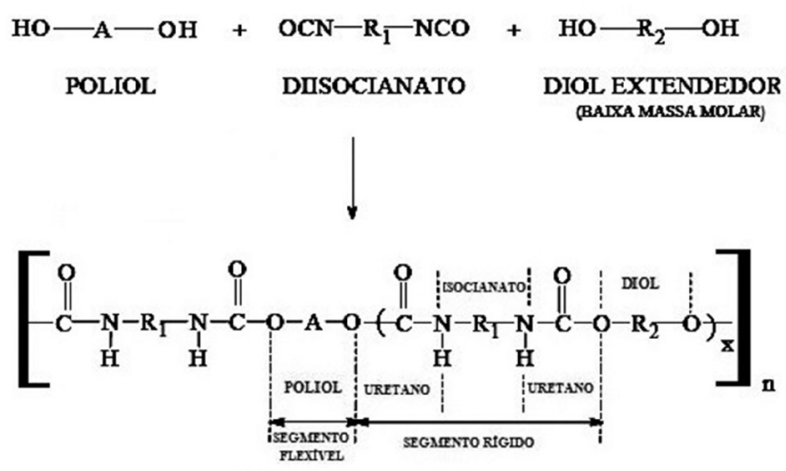

Esquema 2. Obtenção de elastômeros de PU via uma etapa com diol extensor

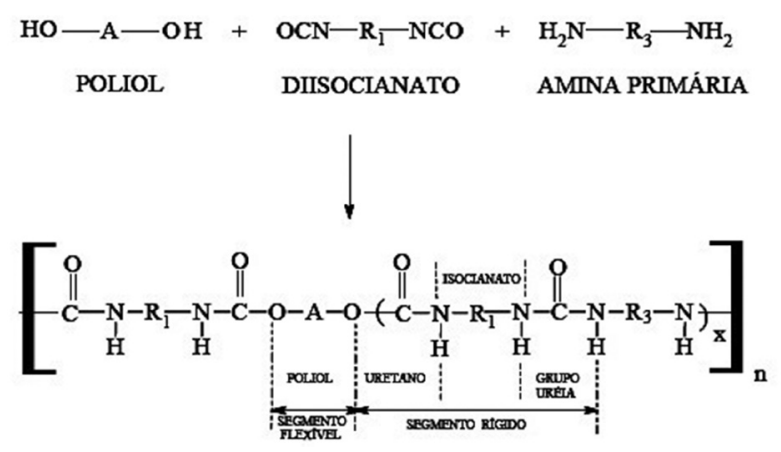

Esquema 3. Copolímero de uretano segmentado com extensão por amina primária

Síntese em uma etapa de binder de óleo de mamona e IPDI (BOMI1 e BOMI2)

Para a síntese de 100 partes do binder BOMI1, foram tratadas 53,00 
partes de óleo de mamona com $99 \%$ de pureza grau PU e valor de hidroxilas $163 \mathrm{mg} \mathrm{KOH} / \mathrm{g}$, durante $3 \mathrm{~h}$ a $120^{\circ} \mathrm{C}$ sob pressão reduzida. Em seguida, foram incorporadas 16,00 partes do plastificante dipropileno glicol dibenzoato (DBDPG, valor de hidroxila $4 \mathrm{mg} \mathrm{KOH} / \mathrm{g}$ ), junto a 4,80 partes de 1,4-BDO e 0,15 partes do catalisador organo-metálico dibutil dilaurato de estanho (DBTD). Após homogeneização dos componentes, acrescentaram-se 26,20 partes de IPDI (teor NCO $=37,50 \%$ ). Em um molde de alumínio, a mistura foi vertida e levada à estufa a $70^{\circ} \mathrm{C}$, curada por $16 \mathrm{~h}$ e desmoldada após esse período.

Empregou-se o mesmo procedimento na síntese de 100 partes do binder BOMI2, porém, com o uso de 41,80 partes de óleo de mamona; 33,50 partes de plastificante DBDPG; 3,80 partes de 1,4 BDO; 21,00 partes de IPDI e 0,15 partes de DBTD.

\section{Síntese em uma etapa de binder de óleo de mamona e Desmo- dur W (BOMW)}

Para a síntese de 100 partes deste binder, 46,80 partes do óleo de mamona foram tratadas durante $3 \mathrm{~h}$ a $120{ }^{\circ} \mathrm{C}$ e, em seguida, incorporam-se 18,70 partes do DBDPG, valor de hidroxila $5 \mathrm{mg}$ $\mathrm{KOH} / \mathrm{g}$, com 4,70 partes de 1,4-butano diol (1,4 BDO), 1,50 partes do antiespumante e 0,50 partes do catalisador DBTD. Os componentes foram homogeneizados a $70{ }^{\circ} \mathrm{C}$ e foram acrescentadas 27,80 partes de Desmodur W (H12MDI com \% NCO =31,80). Realizou-se nova agitação e a mistura foi vertida em moldes de alumínio colocados em estufa a $70{ }^{\circ} \mathrm{C}$, onde permaneceu por $16 \mathrm{~h}$ em processo de cura e desmolde após esse período.

\section{Sistema de pré-polímeros de poliuretano - processo em duas etapas}

Os pré-polímeros são, em geral, líquidos viscosos produzidos pela reação de um di ou poliisocianato com um poliol, formando uma mistura homogênea. Inicialmente, faz-se a adição, a frio, do diisocianato, seguida da adição do poliol nas proporções apropriadas para a obtenção do teor de NCO livre desejado. O teor de NCO livre mostra a porcentagem de [-NCO] em situação reativa na cadeia do pré-polímero, proporcional à sua massa molar total e monitorado através de titulação por dibutilamina (conforme norma ASTM 4274). $\mathrm{O}$ teor de NCO de um pré-polímero se relaciona às propriedades finais do elastômero; por exemplo, em pré-polímeros obtidos a partir de um mesmo diisocianato e poliol, o aumento do teor de NCO livre acarreta um aumento na dureza final do respectivo elastômero.

A reação de obtenção de pré-polímeros é efetuada em atmosfera inerte e em ausência de água, na faixa de temperaturas entre 70-80 ${ }^{\circ} \mathrm{C}$. Acima de $80^{\circ} \mathrm{C}$, podem ocorrer reações secundárias colaterais, como a formação das ligações cruzadas de alofanato e biureto que acarretam o aumento da viscosidade e consequente formação de gel do pré-polímero. O reator empregado é constituído de aço inox 316 , devido a alguns diisocianatos, como o TDI, apresentarem pequenas quantidades de ácido. Durante a reação, deve-se manter a agitação entre 100 e $200 \mathrm{rpm}$. O modelo de reação que ocorre no reator pode ser visto no Esquema 4.

A obtenção dos elastômeros se torna efetiva a partir da reação com um agente de cura, cuja escolha depende dos reagentes empregados e das propriedades desejadas para o produto final. Os mais utilizados são os dióis ou diaminas aromáticas de cadeias curtas. Os dióis, por serem menos reativos que as diaminas, são usados em sistemas mais reativos, como aqueles à base de MDI. Por outro lado, a extensão dos pré-polímeros com diaminas, principalmente em sistemas fabricados com TDI, propicia reações entre uretano e ureia que acarretam propriedades mecânicas importantes para variadas aplicações, tais como: dureza, resistência à tração, baixa deformação permanente $\mathrm{e}$ excelente comportamento dinâmico-mecânico.

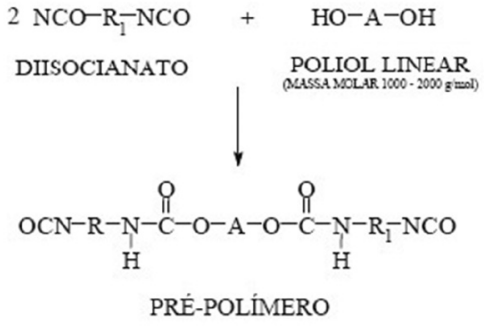

Esquema 4. Obtenção de pré-polímero através da reação de um poliol e diisocianato

Devido ao emprego desses dois tipos de agentes de cura (ou extensores) do pré-polímero, a obtenção de elastômeros de poliuretano via pré-polímeros pode ser efetuada através de duas rotas diferentes, conforme os Esquemas 5 e 6, que mostram a reação utilizando o diol de baixa massa molar e a diamina aromática, respectivamente.
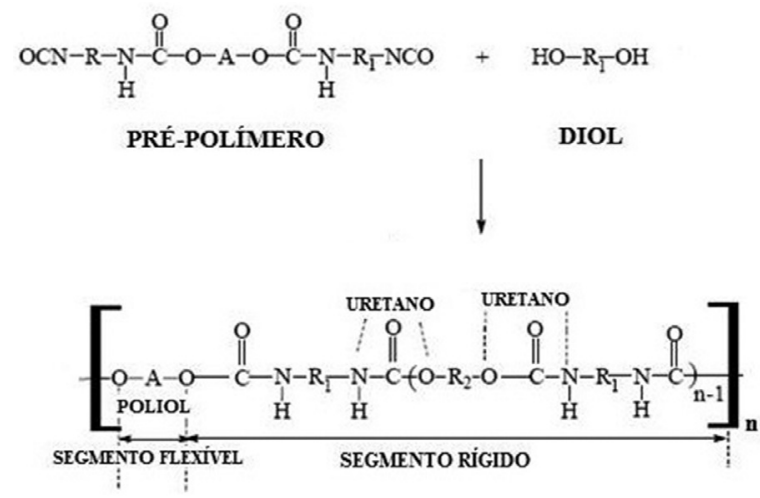

Esquema 5. Obtenção do elastômero via pré-polímeros e um diol de baixa massa molar

O Esquema 6 mostra uma importante rota de obtenção de elastômeros de poliuretano de alto desempenho, pois os grupos ureia formados nesta estrutura propiciam a formação de ligações cruzadas biureto na pós-cura do elastômero por aquecimento. Conforme já dito, essas ligações cruzadas aumentam o módulo e a dureza do poliuretano, proporcionando ao elastômero uma melhoria em suas propriedades mecânicas finais.
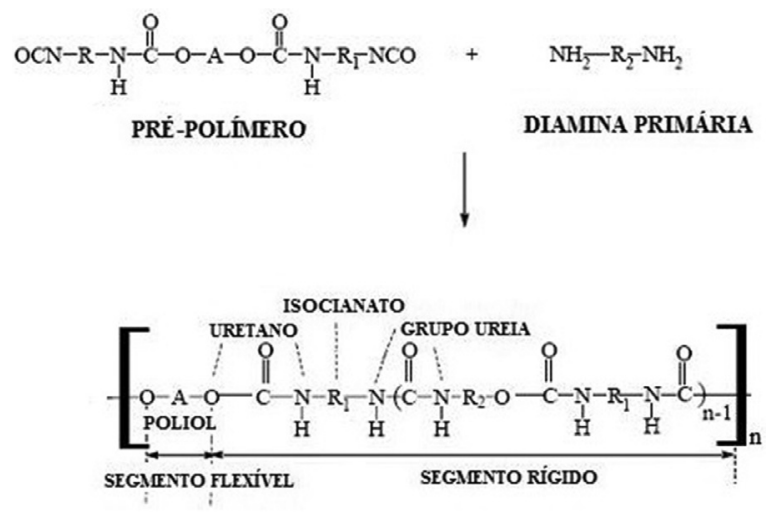

Esquema 6. Obtenção do elastômero de PU via pré-polímero com diamina aromática

Síntese em duas etapas de binders de pré-polímero de uretano com óleo de mamona, PPG e TDI 80/20 (BPG10OMTM e BPG20OMTM)

Para a síntese de 100 partes do binder BPG20OMTM, parte-se do 
pré-polímero com base em óleo de mamona (com 99\% de pureza grau PU, valor de hidroxilas $163 \mathrm{mg} \mathrm{KOH} / \mathrm{g}$ ) e polipropileno glicol (PPG, de nome comercial Voranol® 2120 produzido pela Dow Química). Pesa-se 49,59 partes de Voranol® 2120 e 21,25 partes de óleo de mamona que são, em seguida, misturados e tratados durante $1 \mathrm{~h}$, a $100{ }^{\circ} \mathrm{C}$ sob pressão reduzida. A mistura é resfriada a $50^{\circ} \mathrm{C}$ e adiciona-se 17,67 partes de tolueno diisocianato (TDI 80/20 produzido pela Isopol) sob agitação constante. A temperatura é mantida a $70^{\circ} \mathrm{C}$ sob atmosfera inerte. Após medição do teor de NCO livre por titulação com dibutilamina (4,3\%), o produto é acondicionado em recipiente hermeticamente fechado. O pré-polímero formado é reagido com 11,49 partes de 4,4'-metileno bis-orto-cloroanilina (MOCA $®$ ). Vertese a mistura em moldes de alumínio, seguindo-se à cura em estufa a $70{ }^{\circ} \mathrm{C}$ por $16 \mathrm{~h}$ e desmolde após este período.

A síntese do binder BPG10OMTM, baseado no poliol polipropileno glicol Voranol® 2110 (Dow Química) e óleo de mamona, foi realizada de maneira similar ao BPG20OMTM, utilizando-se 47,0 partes de Voranol® $2110 \mathrm{em}$ vez do Voranol® 2120; 20,20 partes de óleo de mamona, 21,30 partes de TDI 80/20 e 11,50 partes de MOCA®.

\section{Síntese de binder PBLH com Desmodur W e plastificante DBDPG (BPBW)}

Para esta síntese, 72,70 partes do polibutadieno líquido hidroxilado (PBLH, valor típico de hidroxila $45 \mathrm{mg} \mathrm{KOH} / \mathrm{g}$ ) foram tratadas por $3 \mathrm{~h}$ a $100^{\circ} \mathrm{C}$ sob pressão reduzida e, em seguida, incorporaram-se 18,90 partes do plastificante dipropileno glicol dibenzoato (DBDPG, valor de hidroxila $5 \mathrm{mg} \mathrm{KOH} / \mathrm{g}$ ) com 0,50 partes do catalisador organo-metálico dibutil dilaurato de estanho (DBTD). Os componentes foram homogeneizados em um recipiente hermético e, para a obtenção das 100 partes, posteriormente misturados com 7,90 partes do agente de cura Desmodur W (H12MDI, teor de NCO $=31,80 \%$ ). A mistura foi despejada em moldes de alumínio colocados em uma estufa a $70^{\circ} \mathrm{C}$, sendo desmoldada após 16 h de cura.

\section{Obtenção dos combustíveis sólidos ativos CSBOMW e CSPBW}

Também foram realizadas as análises térmicas dos combustíveis sólidos ativos carregados com cargas minerais oxidantes e combustíveis, de forma a comparar o desempenho do binder com base em óleo de mamona ao do binder com base em PBLH.

A Tabela 1 apresenta as formulações dos combustíveis sólidos CSPBW e CSBOMW, desenvolvidos a partir dos binders BPBW (PBLH/Desmodur W) e BOMW (Óleo de Mamona/Desmodur W).

\section{RESULTADOS E DISCUSSÃO}

Para a determinação das temperaturas de transição vítrea $\left(\mathrm{T}_{\mathrm{g}}\right)$ das amostras dos binders obtidos neste trabalho, empregaram-se técnicas de DMTA, DSC, TG e termogravimétrica diferencial (DTG).

\section{Resultados DMTA para os binders baseados em propileno glicol e óleo de mamona (BPG10OMTM e BPG20OMTM)}

Para os binders BPG10OMTM e BPG20OMTM, as curvas dos gráficos DMTA apresentam as variações do módulo de armazenamento (E'), do módulo de perda (E”) e de $\tan \delta$ (razão E"/E') com a temperatura. A temperatura de transição vítrea $\left(\mathrm{T}_{\mathrm{g}}\right)$ foi determinada pelo máximo das curvas de E" em função da temperatura. Os gráficos das Figuras 1 e 2 foram obtidos com uma razão de aquecimento de $3^{\circ} \mathrm{C} / \mathrm{min}$, frequência de $1 \mathrm{~Hz}$ e amplitude de oscilação igual a $0,80 \mathrm{~mm}$.
Tabela 1. Partes em massa dos componentes do combustível sólido CSPBW

\begin{tabular}{llcc}
\hline \multirow{2}{*}{ Componentes } & & CSBOMW & CSPBW \\
\cline { 3 - 4 } & & \multicolumn{2}{c}{$(\%$ em massa $)$} \\
\hline BOMW & Óleo de mamona & 17,97 & - \\
& 1,4 DBO & 1,80 & - \\
& Desmodur W & 12,26 & - \\
BPBW ${ }^{1}$ & PBLH & - & 29,30 \\
& Desmodur W & - & 3,17 \\
DBDPG & & 7,19 & $7,60^{1}$ \\
Perclorato de amônio & & 40,00 & 40,00 \\
Alumínio & & 15,00 & 15,00 \\
Antiespumante & 2,59 & 2,50 \\
Sequestrante de umidade & 3,00 & 2,50 \\
DBTD & & 0,20 & 0,50 \\
Total & & 100 & 100 \\
Agente de ligação (\% sobre o total) & 2 & 2 \\
\hline
\end{tabular}

${ }^{1}$ Para as amostras utilizadas nas análises térmicas, utilizou-se uma formulação contendo DOP como plastificante.

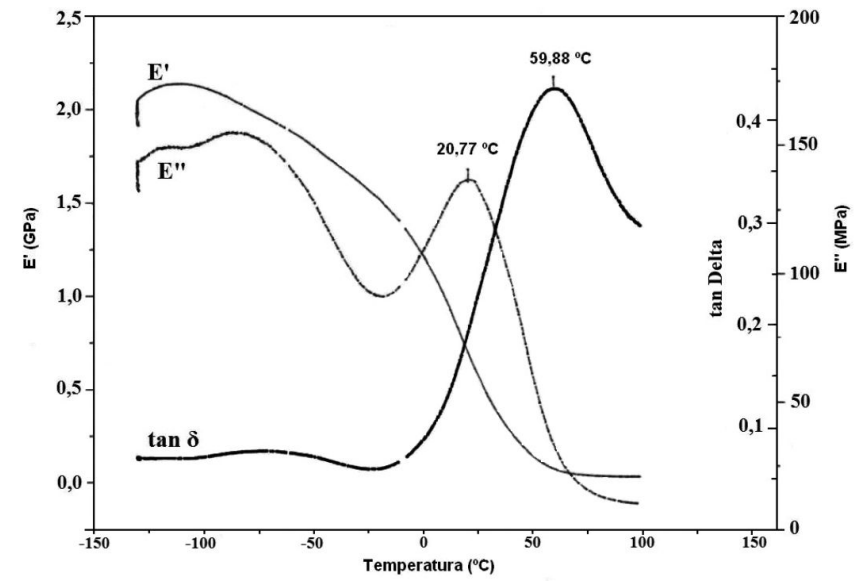

Figura 1. Curvas DMA para o binder BPG20OMTM

A Figura 1 apresenta as curvas DMA para o binder BPG10OMTM. Observando-se a curva para o módulo de amortecimento (E'), seu pico apresenta $\mathrm{T}_{\mathrm{g}}$ de aproximadamente $21^{\circ} \mathrm{C}$. Este resultado era esperado, pois o poliuretano que possui como base o óleo de mamona puro com MDI apresenta $\mathrm{T}_{\mathrm{g}}$ igual a $44{ }^{\circ} \mathrm{C}$. O maior valor na $\mathrm{T}_{\mathrm{g}}$ indica que as cadeias poliméricas perderam mobilidade com a adição de óleo de mamona ao pré-polímero. Desta forma, o óleo de mamona deve ser copolimerizado com um poliol que possua maior mobilidade na cadeia, de forma a aumentar os segmentos flexíveis. Assim, substituiu-se o Voranol 2110 da formulação, cuja massa molar é de $1000 \mathrm{~g} / \mathrm{mol}$, pelo Voranol 2120, de massa molar $2000 \mathrm{~g} / \mathrm{mol}$. A região de transição vítrea se caracteriza pelo aumento no módulo de perda (E”) ou na tangente de perda (tan delta). O módulo de perda (E") apresenta um pico máximo em temperaturas menores do que o pico máximo de atrito interno $(\tan \delta)$. O máximo de dissipação de calor por unidade de deformação ocorre na temperatura em que E" é máximo. Esta temperatura está mais próxima ao valor de temperatura de transição vítrea determinada por outros métodos, como o DSC. Com isso, pode-se utilizar o módulo de perda para se determinar a temperatura e/ou a região de transição vítrea dos materiais poliméricos, ${ }^{24} \mathrm{e}$ a determinação da temperatura de transição vítrea neste trabalho foi realizada adotando-se o máximo do módulo de perda (E”). Com o aumento da massa molar, a mobilidade na cadeia molecular é maior, levando à diminuição da $\mathrm{T}_{\mathrm{g} .}{ }^{5}$ No entanto, observa-se um elevado valor de $\tan \delta$ (aproximadamente $60^{\circ} \mathrm{C}$ ), indicando que o binder considerado não 
tem boa capacidade de recuperação da deformação após a retirada da solicitação térmico-mecânica.

A Figura 2 apresenta as curvas DMA para o binder BPG20OMTM.

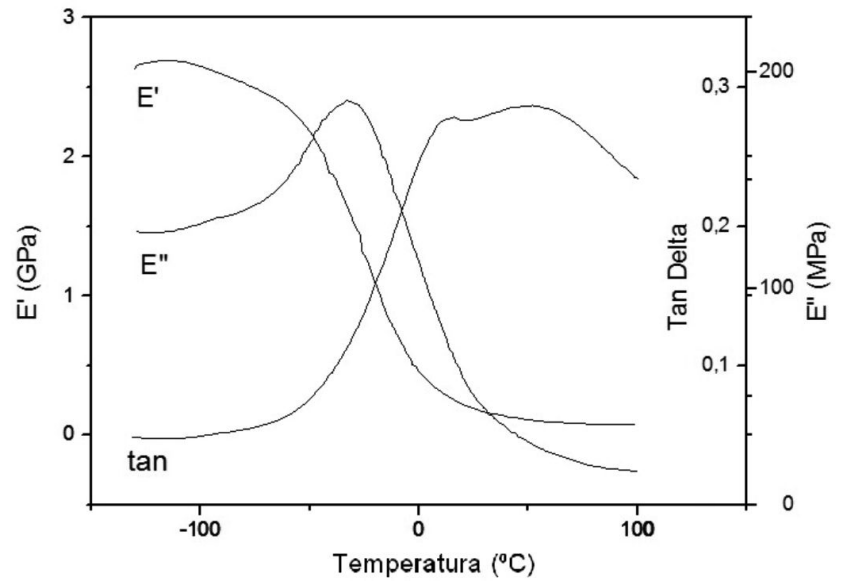

Figura 2. Curvas TG/DTG obtidas para a amostra 1 (sem aditivo)

Nota-se que o pico da curva de E" encontra-se a $-32{ }^{\circ} \mathrm{C}$, o que mostra a esperada redução na $\mathrm{T}_{\mathrm{g}}$, cujo valor para binders e compósitos baseados no polibutadieno líquido hidroxilado $(\mathrm{PBLH})$ varia de $-55 \mathrm{a}-70^{\circ} \mathrm{C}$, segundo a literatura. $\mathrm{O}$ valor de $-32^{\circ} \mathrm{C}$ aproxima-se à faixa de $\mathrm{T}_{\mathrm{g}}$ indicada para binders e compósitos com aplicações em combustíveis e propelentes sólidos, de -40 a $-54{ }^{\circ} \mathrm{C} .{ }^{25,26} \mathrm{Da}$ mesma forma que foi observado para o binder BPG10OMTM, o elevado valor de $\tan \delta\left(52^{\circ} \mathrm{C}\right)$ mostra que o binder BPG20OMTM não tem boa capacidade de recuperação da deformação após a retirada da solicitação térmico-mecânica.

\section{Determinação da temperatura de transição vítrea $\left(\mathrm{T}_{\mathrm{g}}\right)$ por DSC para os binders BOMI, BOMI2 e BOMW}

Amostras dos binders baseados em óleo de mamona e IPDI (BOMI), e em óleo de mamona e Desmodur W (BOMW), foram submetidas à análise DSC, em que o monitoramento é realizado com a variação da entalpia em função da temperatura ou tempo. ${ }^{27}$ Para isso, empregou-se o analisador térmico TA2100 da TA Instruments, módulo DSC-Q910. As corridas foram realizadas com cerca de 10$20 \mathrm{mg}$ de massa de amostra para a determinação da $\mathrm{T}_{\mathrm{g}}$ em função de uma razão programada de aquecimento sob atmosfera de nitrogênio seco, na vazão de $50 \mathrm{~mL} / \mathrm{min}$.

Na Figura 3, estão apresentadas as curvas DSC para os binders BOMI1 e BOMI2.

Observa-se para o binder BOMI1 uma $\mathrm{T}_{\mathrm{g}}$ de, aproximadamente, $-23{ }^{\circ} \mathrm{C}$. Para o binder BOMI2, a curva DSC mostra o valor de $\mathrm{T}_{\mathrm{g}}=$ $-32,7^{\circ} \mathrm{C}$. Esses resultados mostram que ocorre uma sensível queda em relação à $\mathrm{T}_{\mathrm{g}}$ dos binder baseados em óleo de mamona e MDI, que é isento de plastificantes e apresenta o valor de aproximadamente $44^{\circ} \mathrm{C}$. Essa diminuição nas temperaturas de transição vítrea deve-se, provavelmente, à adição do plastificante reativo DBDPG em BOMI1 e BOMI2, que foram formulados com adição de $16,0 \%$ e $33,5 \%$ em massa de DBDPG, respectivamente. Pode-se notar que a diferença na quantidade do plastificante permitiu uma queda de aproximadamente $10^{\circ} \mathrm{C}$ na $\mathrm{T}_{\mathrm{g}}$ do elastômero.

$A T_{g}$ foi considerada como parâmetro decisivo na escolha do agente de cura, o diisocianato, de forma a substituir o IPDI pelo Desmodur W, escolhido por proporcionar elastômeros com propriedades mecânicas diferenciadas, ${ }^{26}$ obtendo-se o binder BOMW. Na Figura 4, apresentam-se suas curvas TG, DSC e DTG.
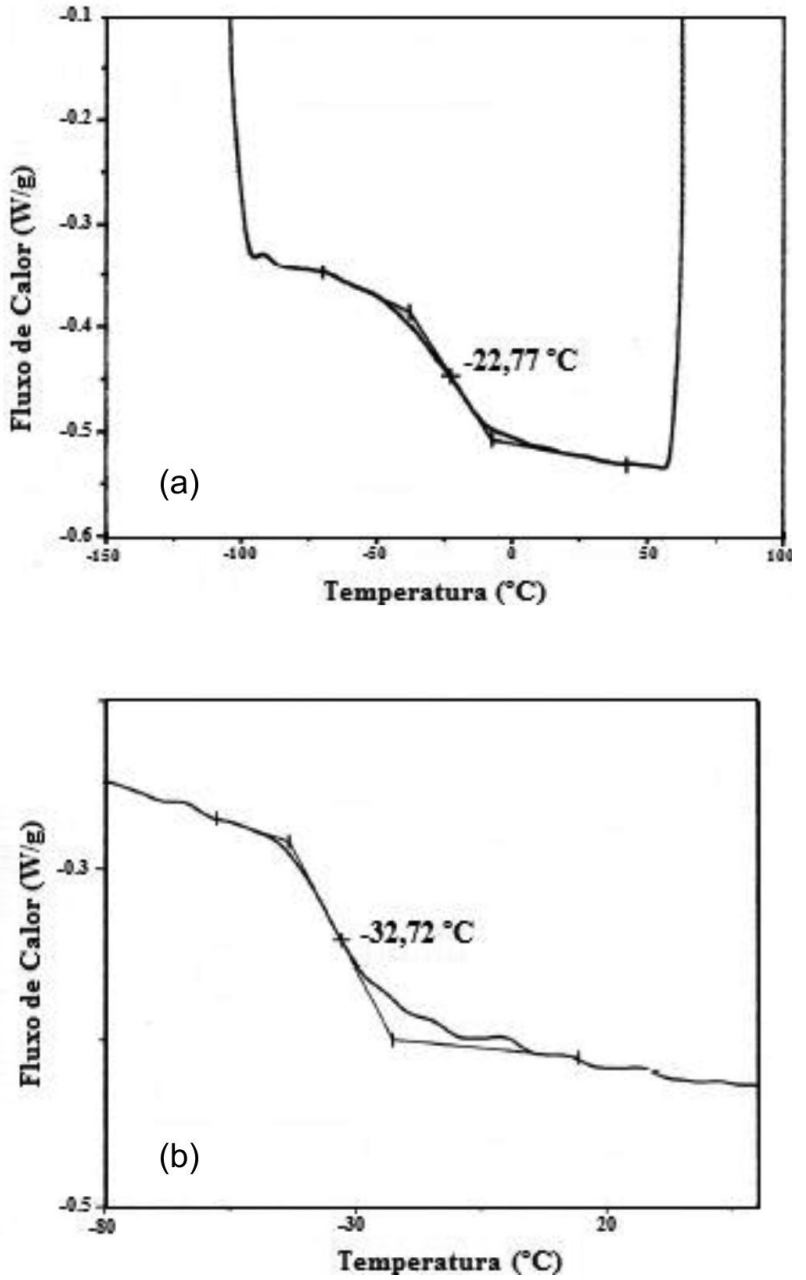

Figura 3. Curvas DSC para a) BOMI1 e b) BOMI2, com razão de aquecimento $10^{\circ} \mathrm{C} / \mathrm{min}$

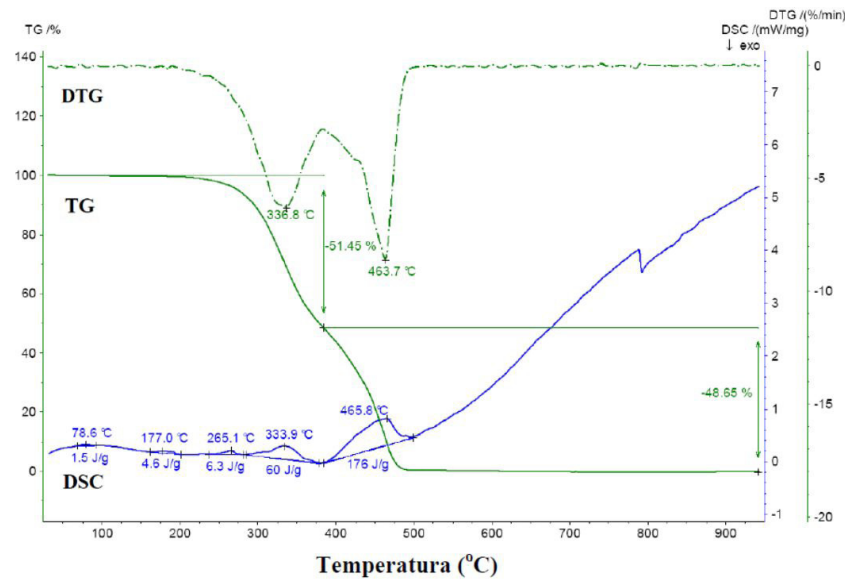

Figura 4. Curvas DSC, TG e DTG para o binder BOMW, na razão de aquecimento de $10^{\circ} \mathrm{C}$ e fluxo de nitrogênio de $50 \mathrm{~mL} / \mathrm{min}$

Foi determinada uma $\mathrm{T}_{\mathrm{g}}$ igual a $-33,5^{\circ} \mathrm{C}$ para o binder $\mathrm{BOMW}$, valor reduzido em relação ao BOMI2. Apesar de essa redução ser pequena, a proporção de plastificante para o BOMW é de 19,05\%, quantidade bastante reduzida em relação ao BOMI2 $(33,45 \%)$, com a vantagem de apresentar propriedades mecânicas superiores.

A curva TG (verde) apresenta uma decomposição térmica em duas etapas, sendo que a primeira ocorre entre 285 a $385{ }^{\circ} \mathrm{C}$, com uma perda de massa de $51,45 \%$ e representa a quebra da cadeia carbônica 
do polímero. A segunda etapa ocorre entre 380 a $485^{\circ} \mathrm{C}$, com uma perda de massa de 48,65\%, e representa a repolimerização parcial das cadeias cuja cisão se deu na primeira etapa. Esse fato é evidenciado pela curva DTG e pode ser explicado pela ocorrência da quebra de ligações cruzadas (decrosslinking). ${ }^{28,29}$

Análises por DSC, TG e DTG para os compósitos com base em óleo de mamona (CSBOMW) e polibutadieno líquido hidroxilado (CSPBW)

Para a determinação da temperatura de transição vítrea do compósito sólido CSBOMW, foi empregada a técnica DSC, cuja curva está representada na Figura 5.

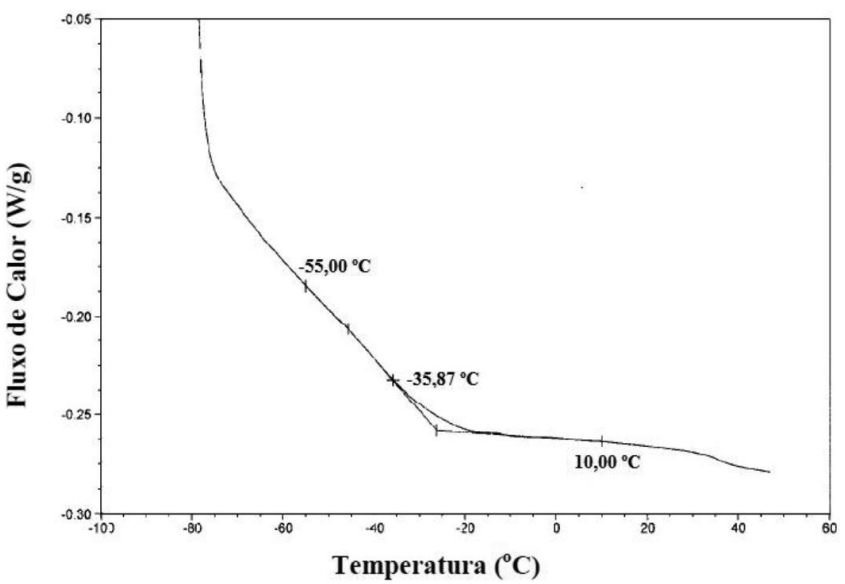

Figura 5. Curva DSC para o compósito combustível sólido CSBOMW, na razão de aquecimento de $10^{\circ} \mathrm{C}$ e fluxo de nitrogênio $50 \mathrm{~mL} / \mathrm{min}$

Observa-se uma $\mathrm{T}_{\mathrm{g}}$ de aproximadamente $-36^{\circ} \mathrm{C}$ para CSBOMW. Esse valor é um pouco mais alto do que o apresentado por compósitos sólidos com base em PBLH (que é de $-55^{\circ} \mathrm{C}$ ), mas próximo da faixa recomendada na literatura, entre $-40 \mathrm{a}-54{ }^{\circ} \mathrm{C} \cdot{ }^{26,30}$

A Figura 6 apresenta as curvas DSC, TG e DTG para a decomposição térmica do compósito combustível sólido CSBOMW, que tem como base o óleo de mamona. Na curva TG, para temperaturas abaixo de $800^{\circ} \mathrm{C}$, são observadas três etapas bem definidas de perdas de massa: de $23,31 \%$ entre 210 a $320{ }^{\circ} \mathrm{C}$; de $48,98 \%$ entre 320 a 430 ${ }^{\circ} \mathrm{C}$; e de $3,79 \%$ entre 490 a $690^{\circ} \mathrm{C}$. A curva mostra que as máximas velocidades de perda de massa ocorrem nas temperaturas de $286^{\circ} \mathrm{C}$, $357^{\circ} \mathrm{C}$ e $394^{\circ} \mathrm{C}$.

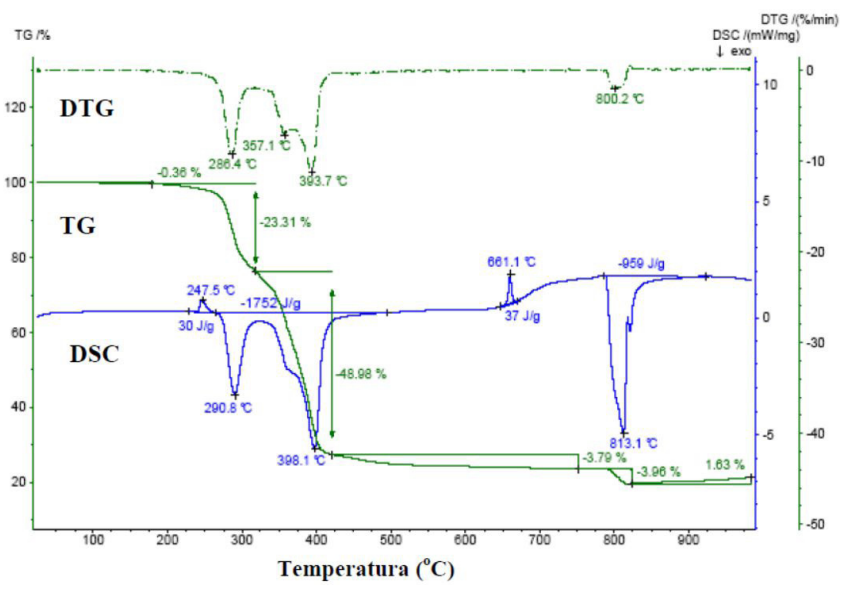

Figura 6. Curvas DTG, TG e DSC para o compósito combustível sólido CS$B O M W$, na razão de aquecimento de $10^{\circ} \mathrm{C}$ e fluxo de nitrogênio de $50 \mathrm{~mL} / \mathrm{min}$
Na curva DSC, durante as etapas de decomposição térmica, observam-se diversos picos endotérmicos e exotérmicos. O pico endotérmico, que ocorre na temperatura de $247,5^{\circ} \mathrm{C}$ está relacionado com a entalpia de $30 \mathrm{~J} / \mathrm{g}$ e indica a mudança na fase cristalina do perclorato de amônio, de monoclínica para cúbica. . $^{13,28,31,32}$

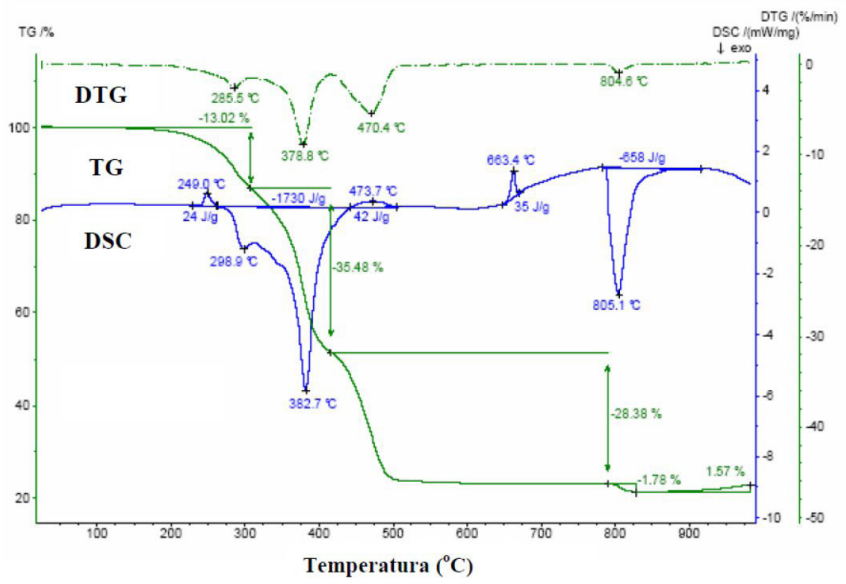

Figura 7. Curvas DTG, TG e DSC para o compósito combustível sólido CS$B P W$, na razão de aquecimento de $10^{\circ} \mathrm{C}$ e fluxo de nitrogênio de $50 \mathrm{~mL} / \mathrm{min}$

Notam-se dois picos exotérmicos da Figura 6 relacionados à entalpia de $-1752 \mathrm{~J} / \mathrm{g}$. O primeiro pico, a $291{ }^{\circ} \mathrm{C}$, provavelmente corresponde ao início da combustão do perclorato de amônio e à combustão dos componentes do binder, tais como o óleo de mamona, o 1,4-butanodiol e o plastificante DBTBD, correspondendo na curva TG a uma perda de massa de $23,31 \%$. Essa perda de massa está relacionada com a saída do plastificante da composição. O segundo pico exotérmico, a $398^{\circ} \mathrm{C}$, corresponde ao final da combustão do perclorato e do binder, relativo a uma perda de massa de 48,93\% demonstrada na curva TG, que ocorre devido à cisão do próprio polímero. Observa-se outro pico endotérmico a $663^{\circ} \mathrm{C}$, com uma entalpia de $35 \mathrm{~J} / \mathrm{g}$. Isso possivelmente ocorre devido à fusão de componentes aditivos do binder, tais como antiespumantes e agente de ligação, que têm como base compostos de silicone e silânicos, correspondendo a uma perda de massa de $-3,79 \%$ apresentada na curva TG com a saída desses aditivos e da carga mineral (quartzo) utilizada no polímero. Após a conexão com o ar atmosférico, a $800{ }^{\circ} \mathrm{C}$, ocorre mais uma etapa de perda de massa de $-3,96 \%$, provavelmente devido à combustão de carbono do aditivo sequestrante de umidade. ${ }^{31-33} \mathrm{O}$ pico exotérmico a $813^{\circ} \mathrm{C}$ na curva DSC reforça esta interpretação.

Na Figura 7, encontram-se as curvas DSC, TG e DTG para amostras do combustível sólido CSPBW, formulado com base em PBLH.

Na curva TG, abaixo da temperatura de $800^{\circ} \mathrm{C}$, é possível observar três etapas principais de perdas de massa: de $13,0 \%$, a cerca de $300{ }^{\circ} \mathrm{C}$, relativo à perda do plastificante; de $35,5 \%$ entre 300 a 400 ${ }^{\circ} \mathrm{C}$ que configura a cisão do polímero; e de $28,4 \%$ entre 400 a $500{ }^{\circ} \mathrm{C}$, devido à saída dos aditivos empregados na composição do binder. Na curva DTG, nota-se que as máximas velocidades de mudança de massa ocorreram nas temperaturas de $286{ }^{\circ} \mathrm{C}, 379{ }^{\circ} \mathrm{C}$ e $470{ }^{\circ} \mathrm{C}$.

Na curva DSC, observam-se diversos eventos ou "picos" endotérmicos e exotérmicos durante as etapas de decomposição térmica das amostras. O pico endotérmico que ocorre a $249^{\circ} \mathrm{C}$ está relacionado à entalpia de $24 \mathrm{~J} / \mathrm{g}$, indicando uma mudança na fase cristalina do perclorato de amônio, de monoclínica para cúbica. ${ }^{13,28}$ Os picos exotérmicos de $249^{\circ} \mathrm{Ce} 383^{\circ} \mathrm{C}$ estão relacionados com a entalpia de $-1730 \mathrm{~J} / \mathrm{g}$. $\mathrm{O}$ primeiro, a $249^{\circ} \mathrm{C}$, deve-se à combustão de componentes do binder tais como o PBLH, Desmodur W e parte do perclorato de amônio. $\mathrm{O}$ segundo pico exotérmico, a $383{ }^{\circ} \mathrm{C}$, corresponde à combustão do 
perclorato de amônio. O pico endotérmico a $474^{\circ} \mathrm{C}$, relativo à entalpia de $42 \mathrm{~J} / \mathrm{g}$, representa a combustão do di-octil ftalato (DOP) utilizado nesta formulação. ${ }^{33}$ Observa-se, com uma entalpia de $35 \mathrm{~J} / \mathrm{g}$, outro pico endotérmico a $663^{\circ} \mathrm{C}$ que provavelmente ocorre devido à fusão de aditivos do binder, tais como antiespumantes e agente de ligação, baseados, respectivamente, em compostos de silicone e silânicos, que correspondem à perda de massa de $1,80 \%$ observada na curva TG. Após conexão com o ar atmosférico, a $800^{\circ} \mathrm{C}$, nota-se mais uma etapa de perda de massa de $1,80 \%$, provavelmente devido à combustão de carbono do aditivo sequestrante de umidade. ${ }^{31-33} \mathrm{O}$ pico exotérmico a $805^{\circ} \mathrm{C}$ na curva DSC vai de acordo com essa informação.

A semelhança entre as curvas DSC e TG dos combustíveis sólidos CSBOMW e CSPBW é um indício de que a combustão de ambos ocorrerá de maneira equivalente, demonstrando a possibilidade de binders modificados com óleo de mamona compor compósitos combustíveis sólidos em substituição àqueles que utilizam o PBLH.

\section{CONCLUSÕES}

Os ensaios iniciais realizados com amostras produzidas com poliuretanos baseados no óleo de mamona mostram a possibilidade de introduzir modificações nos sistemas descritos, de forma a adequá-los ao uso como combustíveis ou propelentes em compósitos sólidos para foguetes e mísseis táticos.

A formulação BOMI1 pôde ser modificada ao alterar o agente de cura de IPDI para Desmodur W, mantendo-se o 1,4-BDO como extensor de cadeia. A simples adição de mais plastificante reativo, conforme a formulação BOMI2, proporcionou uma diminuição significativa na $\mathrm{T}_{\mathrm{g}}$, de $-22^{\circ} \mathrm{C}$ para $-32^{\circ} \mathrm{C}$. Esse baixo valor demonstra que o elastômero é adequado para ser utilizado em condições ambientes, sem que haja perda de suas características elastoméricas.

A decomposição térmica apresentada na curva DSC demonstra picos endotérmicos para a decomposição do binder formulado com base em óleo de mamona BOMW, diferentemente da curva DSC para o binder BPBW, com base em PBLH. Porém, o comportamento das curvas BPBW e CSBOMW se mostrou similar, inclusive os seus valores de entalpias, o que implica dizer que o binder de óleo de mamona, formulado como compósito combustível sólido, apresentará um comportamento em sua combustão similar ao de binders baseados em PBLH.

Desta forma, os resultados mostram que, por meio das modificações propostas, os sistemas de poliuretano com base no óleo de mamona, sejam eles produzidos através do processo one-shot ou com formação de pré-polímero, podem ser empregados como matriz polimérica de propelentes e combustíveis para compósitos sólidos de foguetes e mísseis táticos.

\section{AGRADECIMENTOS}

Os autores agradecem ao CNPq e à AEB pelo apoio financeiro.

\section{REFERÊNCIAS}

1. Akhavan, J.; Polymer 1998, 39, 215.

2. Klager, K.; Polyurethanes, the Most Versatile Binder for Solid Composite Propellants, AIAA/SAE/ASME $20^{\text {th }}$ Joint Propulsion Conference, Ohio, 1984.

3. Vilar, W. D.; Química e Tecnologia de Poliuretanos, Ed. ABPol: São Paulo, 1992
4. Desai, S.; Thakorei, I. M.; Sarawarebe, B. D.; Devi, S.; Eur. Polym. J. 2000, 36, 711 .

5. Scholz, V.; Silva, J. N.; Biomass Bioenergy 2008, 32, 95.

6. Ribeiro, F. J.; Cultura de Mamoneira, Universidade Federal de Viçosa, UFV: Viçosa, 1966.

7. Cangemi, J. M.; Santos, A. M.; Neto, S. C.; Química Nova na Escola 2010, 32, 3 .

8. Belgacem, M. N.; Gandini, A.; Monomers, Polymers and Composites from Renewable Resources; Belgacem, M. N.; Gandini, A., eds.; Elsevier: Amsterdam, 2008.

9. Petrovic Z. S.; Polym. Rev. 2008, 48, 109.

10. Dwivedi, M. C.; Sapre, S.; J. Synth. Lubr. 2002, 19, 229.

11. Mutlu, H.; Meier, M. A. R.; Eur. J. Lipid Sci. Technol. 2010, 112, 10

12. Gunstone, F. D.; The Chemistry of Oil and Fats, $1^{\text {st }}$ ed., Blackwell: New York, 2004.

13. Callister Jr., W. D.; Ciência e Engenharia de Materiais: Uma Introdução, $5^{\text {th }}$ ed., LTC Editora: Rio de Janeiro, 2002.

14. Nacer, R. S.; Poppi, R. R.; Carvalho, P. T. C.; Silva, B. A. K.; Odashiro, A. N.; Silva, I. S.; Delben, J. R. J.; Delben, A. A. S. T.; Acta Cirúrgica Brasileira 2012, 27, 56.

15. Singh, H.; J. Propul. Power 1995, 11, 848.

16. Suarez, P. A. Z.; Meneghetti, S. M. P.; Meneghetti, M. R.; Wolf, C. R.; Quím. Nova 2007, 30, 667.

17. Weiss, E.A.; Oilseed Crops, Longman: London, 1983.

18. Bazaki, H.; Kubota, N.; Propellants, Explos., Pyrotech. 2000, 25, 312.

19. Stankovic, M.; Kapor, V.; Petrovic, S.; J. Therm. Anal. Calorim. 1999, 56, 1383.

20. Jones, D. E. G.; Feng, H. T.; Augsten, R. A.; Fouchard, R. C.; J. Therm. Anal. Calorim. 1999, 55, 9.

21. Lourenço, V. L.; Introdução à Análise Térmica, CTA-IAE-Divisão de Química: São José dos Campos, 1999.

22. Rocco, J. A. F. F.; Lima, J. E. S.; Lourenço, V. L.; Batista, N. L.; Botelho, E. C.; Iha, K.; J. Appl. Polym. Sci. 2012, 126, 1461.

23. Kwon, S. J.; Kim, T. Y.; Lee, B. S.; Lee, T. H.; Kim, J. E.; Suh, K. S.; Synth. Met. 2010, 160, 1092.

24. Murayama, T.; Dynamic Mechanical Analysis of Polymeric Material, Elsevier Scientific Publishing Company: Amsterdam, 1982.

25. Sutton, G. P.; Rocket Propulsion Elements, $7^{\text {th }}$ ed., Wiley \& Sons: New York, 2001.

26. Winborg, N.; Tese de Doutorado, Royal Technology Institute of Stockholm, Suécia, 2004.

27. Andrade, J.; Iha, K.; Rocco, J. A. F. F.; Bezerra, E. M.; Suarez-Iha, M. E. V.; Pinheiro, G. F. M.; Quím. Nova 2007, 30, 952.

28. Brill, T. B.; Budenz, B. T.; Flash Pyrolysis of Ammonium Perchlorate Hydroxyl-Terminated-Polybutadiene Mixtures Including Selected Additives, American Institute of Aeronautics and Astronautics, Charles Stark Draper Laboratory, Inc.: Massachusetts, 2000.

29. Prashantha, K.; Pai, V. K.; Sherigara, B. S.; Prasannakumar, S.; Bull. Mater. Sci. 2001, 24, 535.

30. Cohen, N. S.; Fleming, R. W.; Derr, R. L.; AIAA J. 1974, 12, 212.

31. Rocco, J. A. F. F.; Lima, J. E. S.; Iha, K.; Frutuoso, A. G.; Suárez-Iha, M. E. V.; Ionashiro, M.; Matos, J. R.; J. Therm. Anal. Calorim. 2004, 75,551 .

32. Rocco, J. A. F. F.; Lima, J. E. S.; Iha, K.; Frutuoso, A. G.; Suárez-Iha, M. E. V.; Ionashiro, M.; Matos, J. R.; J. Therm. Anal. Calorim. 2004, 77,803 .

33. Lu, Y. C.; Kuo, K. K.; Thermochim. Acta 1996, 275, 181. 